\title{
The Nodal Cubic is a Quantum Homogeneous Space
}

\author{
Ulrich Krähmer $^{1}$ • Angela Ankomaah Tabiri ${ }^{1}$
}

Received: 5 September 2016 / Accepted: 4 November 2016 / Published online: 7 January 2017

(C) The Author(s) 2017. This article is published with open access at Springerlink.com

\begin{abstract}
The cusp was recently shown to admit the structure of a quantum homogeneous space, that is, its coordinate ring $B$ can be embedded as a right coideal subalgebra into a Hopf algebra $A$ such that $A$ is faithfully flat as a $B$-module. In the present article such a Hopf algebra $A$ is constructed for the coordinate ring $B$ of the nodal cubic, thus further motivating the question which affine varieties are quantum homogeneous spaces.
\end{abstract}

Keywords Hopf algebra - Quantum homogeneous space · Singular curve · Noncommutative Galois extension

Mathematics Subject Classification (2010) $16 \mathrm{~T} 05 \cdot 16 \mathrm{~T} 20 \cdot 14 \mathrm{H} 50$

Just as quantum groups (Hopf algebras) generalise affine algebraic groups, quantum homogeneous spaces as studied e.g. in $[2,7,11,14-19,21]$ generalise affine varieties with a transitive action of an algebraic group:

Definition A quantum homogeneous space is a right coideal subalgebra $B$ of a Hopf algebra $A$ which is faithfully flat as a left $B$-module.

There is also an analytic theory of transitive or more generally ergodic actions of compact or locally compact quantum groups, see e.g. $[8,13]$ and the references therein.

Presented by Paul Smith.

Ulrich Krähmer

ulrich.kraehmer@glasgow.ac.uk

Angela Ankomaah Tabiri

a.tabiri.1@ research.gla.ac.uk

1 School of Mathematics and Statistics, 15 University Gardens, University of Glasgow, Glasgow, G12 8QW, UK 
The best studied examples are deformation quantisations of affine homogeneous spaces, and in particular Podleś' quantum 2-spheres [20]. However, even if $A$ is noncommutative, $B$ can be a commutative algebra, so a natural question to ask is:

\section{Question Which affine varieties are quantum homogeneous spaces?}

In the operator algebraic setting, the analogue of this question has been raised and studied for example in [12]. Here we consider the purely algebraic setting, working over an algebraically closed field $k$ of characteristic 0 .

Unlike a homogeneous space, an affine variety which is a quantum homogeneous space can be singular as the example of the cusp shows (see [15, Section 2.11] and [10, Construction 1.2]). Note that by the results from [9], noncommutative Hopf algebras coacting on commutative algebras are quite restricted. Still, we conjecture that every plane curve can be given the structure of a quantum homogeneous space, and our aim here is to point this out for the nodal cubic given by the equation $y^{2}=x^{2}+x^{3}$ :

Theorem Fix $(q, p) \in \kappa^{2}$ satisfying $p^{2}=q^{2}+q^{3}$. Then the unital associative $\kappa$-algebra $A$ with generators $x, y, a, a^{-1}, b$ satisfying the relations

$$
\begin{gathered}
a a^{-1}=a^{-1} a=1, \quad y^{2}=x^{2}+x^{3}, \quad b^{2}=a^{3}, \\
y a=a b, \quad b x=x b, \quad y x=x y, \quad b y=-y b+2 p b^{2}, \\
a^{2} x=-x a^{2}-a x a-a^{2}+(1+3 q) a^{3}, \\
a x^{2}=-a x-x a-x^{2} a-x a x+(2+3 q) q a^{3} .
\end{gathered}
$$

admits a Hopf algebra structure whose coproduct $\Delta$, counit $\varepsilon$ and antipode $S$ satisfy

$$
\begin{array}{r}
\Delta(x)=1 \otimes(x-q a)+x \otimes a, \quad \Delta(y)=1 \otimes(y-p b)+y \otimes b, \\
\Delta(a)=a \otimes a, \quad \Delta(b)=b \otimes b, \quad \varepsilon(x)=q, \quad \varepsilon(y)=p, \quad \varepsilon(a)=\varepsilon(b)=1, \\
S(x)=q-(x-q) a^{-1}, \quad S(y)=p-(y-p) b^{-1}, \quad S(a)=a^{-1}, \quad S(b)=b^{-1} .
\end{array}
$$

Furthermore, the right coideal subalgebra $B \subset A$ generated by $x, y$ is the coordinate ring of the nodal cubic, and $A$ is free and in particular faithfully flat as a B-module.

Observe that the commutation relations in $A$ are chosen in such a way that

$$
(y-p b)^{2}=y^{2}-p^{2} b^{2}, \quad(x-q a)^{2}+(x-q a)^{3}=x^{2}+x^{3}-\left(q^{2}+q^{3}\right) a^{3}
$$

so that

$$
(y-p b)^{2}=(x-q a)^{2}+(x-q a)^{3} .
$$

Thus informally speaking each coordinate on the curve becomes perturbed by some additional group-like "quantum coordinate" and the perturbed coordinates still satisfy the defining relation of the curve. As $x-q a$ and $y-p b$ are twisted primitive, the Hopf algebra $A$ is generated by group-likes and twisted primitives. This implies:

\section{Proposition The Hopf algebra A is pointed.}

The point $(q, p)$ on the curve is the point the quantum orbit of which it is presented as. To use these observations as starting point of a general study of quantum homogeneous space structures on affine varieties seems a promising future research direction. 
The proof of the theorem consists of a straightforward (albeit tedious) verification that the formulas for the coproduct, counit and antipode are compatible with the defining relations of $A$, followed by a similarly straigthforward application of Bergman's diamond lemma [1] yielding a vector space basis of $A$ that implies the freeness over $B$ :

\section{Proposition The set}

$$
\left\{x^{i} y^{j}(a x)^{l} a^{m} b^{n} \mid i, l \in \mathbb{N}, j \in\{0,1\}, m \in \mathbb{Z}, n \in\{0,1\}\right\}
$$

is a vector space basis of $A$, and the GK-dimension of A equals 3.

Using this basis, one also easily observes that like the nonstandard Podleś spheres, the algebra extension $B \subset A$ is an example of a coalgebra Galois extension [3-6] rather than of a Hopf-Galois extension: the coalgebra is $C:=A / B^{+} A$, where $B^{+}:=B \cap \operatorname{ker} \varepsilon$. The canonical projection $\pi: A \rightarrow C$ defines a left $C$-coaction

$$
\lambda: A \rightarrow C \otimes A, \quad f \mapsto f_{(-1)} \otimes f_{(0)}:=\pi\left(f_{(1)}\right) \otimes f_{(2)}
$$

and we have (as a consequence of the faithful flatness of $A$ over $B$ )

$$
B=\left\{f \in A \mid f_{(-1)} \otimes f_{(0)}=\pi(1) \otimes f\right\} .
$$

That $C$ is not a Hopf algebra quotient of $A$ follows from $B^{+} A \neq A B^{+}$ (cf. [18, Lemma 1.4]); for example, we have

$$
A B^{+} \ni a^{2}(x-q)=-x a^{2}-a x a-(1+q) a^{2}+(1+3 q) a^{3} \notin B^{+} A .
$$

We finally remark that some properties of the algebra $A$ are better understood when using a slightly different set of generators: if we abbreviate

$$
c:=3 x-(1+3 q) a+1, \quad d:=3 y-6 p b, \quad e:=a c+r c a
$$

where $r$ is a primitive 6 th root of 1 (so that $r+r^{-1}=1$ ), then the defining relations of $A$ in terms of the generators $a^{ \pm 1}, b, c, d, e$ read

$$
\begin{gathered}
a a^{-1}=a^{-1} a=1, \quad a b=b a, \quad a c+r c a=e, \quad a d=d a, \quad a e+r^{-1} e a=0, \\
b c=c b, \quad b d=-d b, \quad b e=e b, \quad b^{2}=a^{3}, \quad c d=d c, \quad r^{-1} c e+e c=3\left(a-a^{3}\right), \\
d e=e d, \quad 3 d^{2}=c^{3}-3 c+2+(1+3 q)\left(-2+6 q+9 q^{2}\right) a^{3} .
\end{gathered}
$$

Using these generators, one easily verifies for example:

Proposition The units in $A$ are of the form $\alpha a^{i} b^{j}, \alpha \in k, i \in \mathbb{Z}, j \in\{0,1\}$.

Acknowledgments We thank Ken Brown and Tomasz Brzeziński for helpful comments on a first draft of this paper. A.T. is funded by a Faculty for the Future Fellowship of the Schlumberger Foundation.

Open Access This article is distributed under the terms of the Creative Commons Attribution 4.0 International License (http://creativecommons.org/licenses/by/4.0/), which permits unrestricted use, distribution, and reproduction in any medium, provided you give appropriate credit to the original author(s) and the source, provide a link to the Creative Commons license, and indicate if changes were made.

\section{References}

1. Bergman, G.M.: The diamond lemma for ring theory. Adv. in Math. 29(2), 178-218 (1978). doi:10.1016/0001-8708(78)90010-5. MR506890 
2. Brown, K., Gilmartin, P.: Quantum homogeneous spaces of connected hopf algebras. J. Algebra 454, 400-432 (2016). doi:10.1016/j.jalgebra.2016.01.030. MR3473433

3. Brzeziński, T.: On modules associated to coalgebra galois extensions. J. Algebra 215(1), 290-317 (1999). doi:10.1006/jabr.1998.7738. MR1684158

4. Brzeziński, T., Hajac, P.M.: Coalgebra extensions and algebra coextensions of galois type. Comm. Algebra 27(3), 1347-1367 (1999). doi:10.1080/00927879908826498. MR1669095

5. Brzeziński, T., Hajac, P.M.: The chern-galois character. English, with English and French summaries. C. R. Math. Acad. Sci. Paris 338(2), 113-116 (2004). doi:10.1016/j.crma.2003.11.009. MR2038278

6. Brzeziński, T., Majid, S.: Coalgebra bundles. Comm. Math. Phys. 191(2), 467-492 (1998). doi:10.1007/s002200050274. MR1604340

7. De Commer, K.: On the construction of quantum homogeneous spaces from *-galois objects. Algebr. Represent. Theory 15(4), 795-815 (2012). doi:10.1007/s10468-011-9265-7. MR2944442

8. De Commer, K., Yamashita, M.: Tannaka-krein duality for compact quantum homogeneous spaces. i. General theory. Theory Appl. Categ. 28(31), 1099-1138 (2013). MR3121622

9. Etingof, P., Debashish, G., Mandal, A., Walton, C.: Hopf coactions on commutative algebras generated by a quadratically independent comodule. Comm. Algebra. to appear

10. Goodearl, K.R., Zhang, J.J.: Noetherian hopf algebra domains of gelfand-kirillov dimension two. J. Algebra 324(11), 3131-3168 (2010). doi:10.1016/j.jalgebra.2009.11.001. MR2732991

11. Heckenberger, I., Kolb, S.: Differential calculus on quantum homogeneous spaces. Lett. Math. Phys. 63(3), 255-264 (2003). doi:10.1023/A:1024496304742. MR1992890

12. Huang, H.: Faithful compact quantum group actions on connected compact metrizable spaces. J. Geom. Phys. 70, 232-236 (2013). doi:10.1016/j.geomphys.2013.03.027. MR3054297

13. Kasprzak, P., Sołtan, P.M.: Embeddable quantum homogeneous spaces. J. Math. Anal. Appl. 411(2), 574-591 (2014). doi:10.1016/j.jmaa.2013.07.084. MR3128415

14. Kolb, S., Pellegrini, J.: Braid group actions on coideal subalgebras of quantized enveloping algebras. J. Algebra 336, 395-416 (2011). doi:10.1016/j.jalgebra.2011.04.001. MR2802552

15. Krähmer, U.: On the hochschild (co)homology of quantum homogeneous spaces. Israel J. Math. 189, 237-266 (2012). doi:10.1007/s11856-011-0168-4. MR2931396

16. Liu, L., Wu, Q.: Twisted calabi-yau property of right coideal subalgebras of quantized enveloping algebras. J. Algebra 399, 1073-1085 (2014). doi:10.1016/j.jalgebra.2013.10.019. MR3144627

17. Liu, L., Wu, Q.: Rigid dualizing complexes over quantum homogeneous spaces. J. Algebra 353, 121-141 (2012). doi:10.1016/j.jalgebra.2011.12.007. MR2872439

18. Müller, E.F., Schneider, H.-J.: Quantum homogeneous spaces with faithfully flat module structures. Israel J. Math. 111, 157-190 (1999). doi:10.1007/BF02810683. MR1710737

19. Ó Buachalla, R.: Noncommutative complex structures on quantum homogeneous spaces. J. Geom. Phys. 99, 154-173 (2016). doi:10.1016/j.geomphys.2015.10.003. MR3428362

20. Podleś, P.: Quantum spheres. Lett. Math. Phys. 14(3), 193-202 (1987). doi:10.1007/BF00416848. MR919322

21. Schneider, H.-J.: Principal homogeneous spaces for arbitrary hopf algebras Hopf algebras. Israel J. Math. 72(1-2), 167-195 (1990). doi:10.1007/BF02764619. MR1098988 